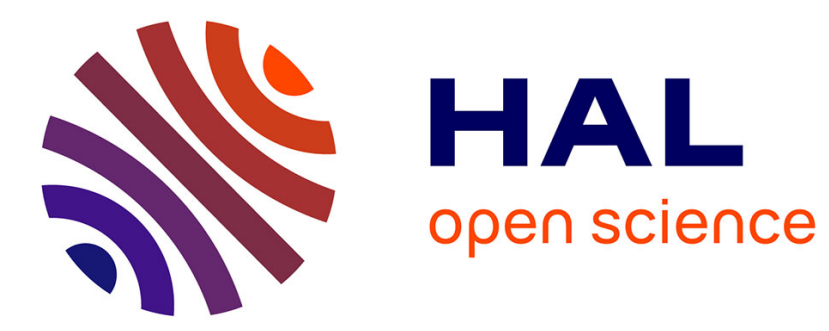

\title{
Ensemble pour spectrométrie Môssbauer haute température in situ. Evolution thermique des paramètres Môssbauer de $\mathrm{SrFeO2,50}$
}

\author{
L. Fournes, Y. Potin, J.C. Grenier, P. Hagenmuller
}

\section{- To cite this version:}

L. Fournes, Y. Potin, J.C. Grenier, P. Hagenmuller. Ensemble pour spectrométrie Môssbauer haute température in situ. Evolution thermique des paramètres Môssbauer de $\mathrm{SrFeO} 2,50$. Revue de Physique Appliquée, 1989, 24 (4), pp.463-468. 10.1051/rphysap:01989002404046300 . jpa-00246068

\section{HAL Id: jpa-00246068 https://hal.science/jpa-00246068}

Submitted on 1 Jan 1989

HAL is a multi-disciplinary open access archive for the deposit and dissemination of scientific research documents, whether they are published or not. The documents may come from teaching and research institutions in France or abroad, or from public or private research centers.
L'archive ouverte pluridisciplinaire HAL, est destinée au dépôt et à la diffusion de documents scientifiques de niveau recherche, publiés ou non, émanant des établissements d'enseignement et de recherche français ou étrangers, des laboratoires publics ou privés. 


\title{
Ensemble pour spectrométrie Mössbauer haute température in situ. Evolution thermique des paramètres Mössbauer de $\mathrm{SrFeO}_{2,50}$
}

\author{
L. Fournes, Y. Potin, J. C. Grenier et P. Hagenmuller \\ Laboratoire de Chimie du Solide du CNRS, Université de Bordeaux I, 351 cours de la Libération, \\ 33405 Talence Cedex, France
}

(Reçu le 4 décembre 1988, révisé le 12 décembre 1988, accepté le 5 janvier 1989)

\begin{abstract}
Résumé. - Une cellule pour l'étude in situ de la résonance Mössbauer est décrite. Elle est conçue pour des mesures jusqu'à $1100 \mathrm{~K}$ dans un domaine de pression gazeuse allant de $1 \mathrm{~Pa}$ à $2 \times 10^{5} \mathrm{~Pa}$. L'échantillon pulvérulent est déposé sur un disque en nitrure de bore. L'ensemble est placé au centre du réacteur. La cellule a été utilisée pour l'étude du comportement thermique de $\mathrm{SrFeO}_{2,50}$.
\end{abstract}

\begin{abstract}
An in situ cell for Mössbauer resonance studies has been described. It allows measurements up to $1100 \mathrm{~K}$ with a gas pressure ranging from $1 \mathrm{~Pa}$ to $2 \times 10^{5} \mathrm{~Pa}$. The finely powdered sample is layed on a boron nitride holder. The whole is placed at the center of the reactor. The cell has been used to determine the thermal behavior of $\mathrm{SrFeO}_{2.50}$.
\end{abstract}

\section{Introduction.}

Divers modèles de cellules pour des études par spectroscopie Mössbauer de réactions solide-gaz ont été proposés. Dans la majorité des cas celles-ci mettent en cuvre un absorbant en position verticale [1-4]. Un tel dispositif implique soit la mise en place de l'échantillon entre deux fines feuilles métalliques soit l'addition d'un liant. Dans les deux cas la réactivité de l'échantillon vis-à-vis de son atmosphère s'en trouve considérablement diminuée. Certains auteurs ont proposé cependant des cellules dans lesquelles l'échantillon est en position horizontale [5-9]. Dans ces conditions ce dernier doit être pastillé et la réaction n'a lieu souvent que pour de faibles épaisseurs avec apparition d'un gradient de concentration.

Notre objectif était d'utiliser des échantillons minces pulvérulents déposés sur support horizontal inerte. Un appareil a donc été construit, à savoir un réacteur contenant un microfour porte-échantillon et permettant une circulation de gaz. Son encombrement a été réduit au maximum pour obtenir une absorption aussi faible que possible des rayons $\gamma$. Les matériaux utilisés sur le trajet du rayonnement ont été choisis aussi peu absorbants que possible.
Le système $\mathrm{SrFeO}_{3-y}$ est maintenant bien connu pour son domaine d'existence étendu $(0 \leqslant y \leqslant 0,50)$ et pour la présence du fer à deux degrés d'oxydation différents [10-18]. Le taux de lacunes dépend de la température et de la pression d'oxygène [16]. Des études par résonance Mössbauer in situ étaient donc particulièrement judicieuses.

Nous avons illustré l'emploi d'une nouvelle cellule par l'étude de l'évolution thermique des paramètres Mössbauer de la phase de type brownmillérite $\mathrm{SrFeO}_{2,50}(y=0,50)$ qui est particulièrement réactive. Une détermination des caractéristiques Mössbauer à basse température a également été effectuée pour compléter cette étude.

\section{Réalisation d'un ensemble pour spectrométrie} Mössbauer in situ de haute température.

2.1 DESCRIPTION DU RÉACTEUR. - Le réacteur est représenté à la figure 1 . Il est constitué de deux tubes de quartz soudés formant une croix (a). Le quartz a été choisi pour sa bonne tenue en température et sa faible conductibilité thermique longitudinale. L'isolation thermique du réacteur est obtenue grâce à l'emploi de laine de roche (b). 


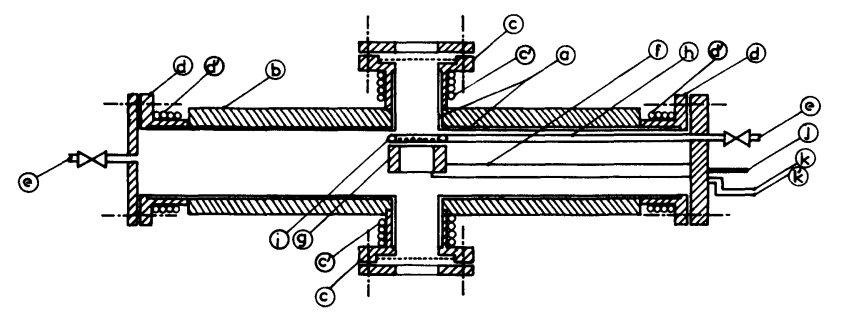

Fig. 1. - Schéma général du réacteur.

[General diagram of the reactor.]

Des brides en acier inoxydable sont collées aux extrémités des tubes de quartz (c, d). Sur ces quatre brides sont brasés des enroulements en cuivre permettant un refroidissement efficace par circulation d'eau $\left(c^{\prime}, d^{\prime}\right)$.

Les deux brides de type (c) permettent la mise en place des fenêtres dont le montage est donné à la figure 2. Les fenêtres (m) sont collées dans un porte-

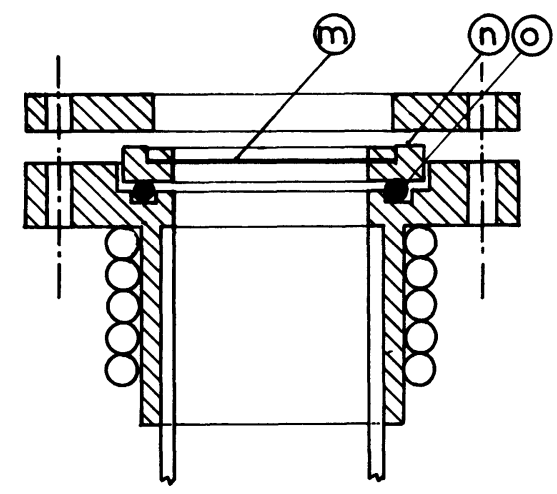

Fig. 2. - Schéma détaillé de la bride porte-fenêtre.

[Detailed diagram of the clamp for the window.]

fenêtre (n). L'étanchéité est assurée par un joint torique (o). Deux types de fenêtres ont été utilisés :

- des fenêtres en composite aluminium-mylar résistant aux atmosphères oxydantes et destinées aux études effectuées en dessous de $800 \mathrm{~K}$;

- des fenêtres en aluminium ultra-pur d'épaisseur $25 \mu \mathrm{m}$. Celles-ci sont employées lorsqu'on impose au microfour des températures supérieures à $800 \mathrm{~K}$.

Sur les deux autres brides (type d, Fig. 1) sont soudées les vannes d'entrée et sortie des gaz (e). Sur une de ces deux brides est fixé le bras (f) qui supporte l'ensemble microfour-porte-échantillon (g) ainsi que le tube d'arrivée des gaz (h). Celui-ci est terminé par une couronne percée de trous (i) à proximité de l'échantillon. Lorsque le débit des gaz entrant dans la cellule est suffisamment faible, il n'est pas nécessaire de les préchauffer avant leur introduction. Des passages étanches sont prévus pour l'élément chauffant $(\mathrm{j})$ et les thermocouples $(\mathrm{k}$ et $\mathrm{k}^{\prime}$ ).

2.2 ENSEMBLE MICROFOUR-PORTE-ÉCHANTILLON. - Le schéma détaillé du microfour-porte-échantillon et de sa bride support est représenté à la figure 3 .

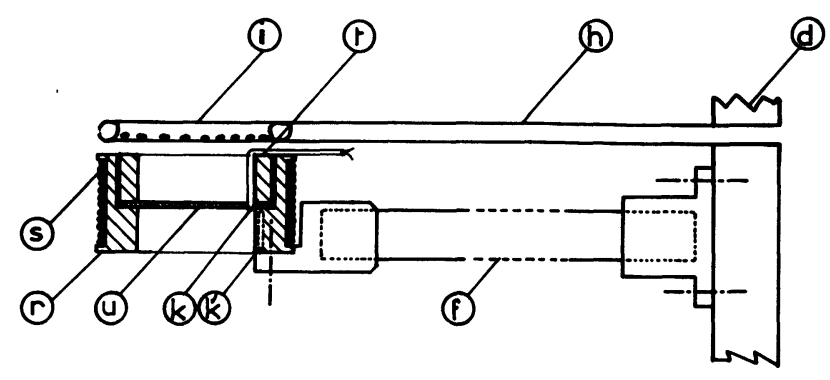

Fig. 3. - Schéma détaillé de l'ensemble microfour porteéchantillon.

[Detailed diagram of the microfurnace and of the sample holder.]

Le microfour est constitué d'un cylindre métallique rainuré (r) sur lequel est enroulé l'élément chauffant de type thermocoax (s). Cet ensemble permet un bon transfert thermique avec le support et on peut atteindre ainsi des températures de l'ordre de $1100 \mathrm{~K}$. La position du microfour sur le bras (f) et la fixation du bras sur la bride (d) permettent un alignement parfait du microfour avec les fenêtres du réacteur. Une bague ( $t$ ) sert à bloquer le porteéchantillon (u).

Le porte-échantillon en forme de disque doit être constitué d'un matériau chimiquement inerte. Divers matériaux ont été testés. Les meilleurs résultats ont été obtenus avec le nitrure de bore. Le porteéchantillon est une pastille de $32 \mathrm{~mm}$ de diamètre et de $1,5 \mathrm{~mm}$ d'épaisseur préparée sous une pression de $2 \mathrm{t} / \mathrm{cm}^{2}$ à partir de nitrure de bore ultra-pur, pulvérulent et très sec. Cette pastille est changée à chaque étude pour éviter toute contamination des échantillons.

Celui-ci sous forme pulvérulente est déposé sur le porte-échantillon de façon homogène à l'aide d'un vibreur électrique. L'épaisseur du dépôt (absorbeur), très faible $(\simeq 100 \mu \mathrm{m})$, limite les risques d'établissement en son sein d'un gradient de température ; il permet une réaction homogène de l'échantillon avec le gaz.

2.3 CONDITIONS DE FONCTIONNEMENT. - Un important problème en spectrométrie Mössbauer est la connaissance exacte de la température de l'échantillon. La plupart des montages proposés antérieurement ne comportent qu'un seul thermocouple de contrôle au sein du porte-échantillon. Les auteurs supposaient qu'il n'existait aucun gradient de température entre l'absorbeur et le porte-échantillon. Ceci 
est certainement d'autant moins vrai que les études étaient effectuées à des températures élevées. Pour pallier cet inconvénient nous utilisons deux thermocouples chromel-alumel :

- un thermocouple noyé dans la masse du microfour (Fig. 3-k), dont la soudure chaude se trouve aussi proche que possible de l'échantillon. Ce thermocouple sert de sonde pour la régulation;

- un second thermocouple (Fig. 3-k') qui vient se loger dans l'épaisseur du porte-échantillon en nitrure de bore.

Le schéma du système de contrôle et de régulation de la température est donné à la figure 4. La variation de température au cours de l'accumulation des données de résonance Mössbauer n'excède pas un degré.

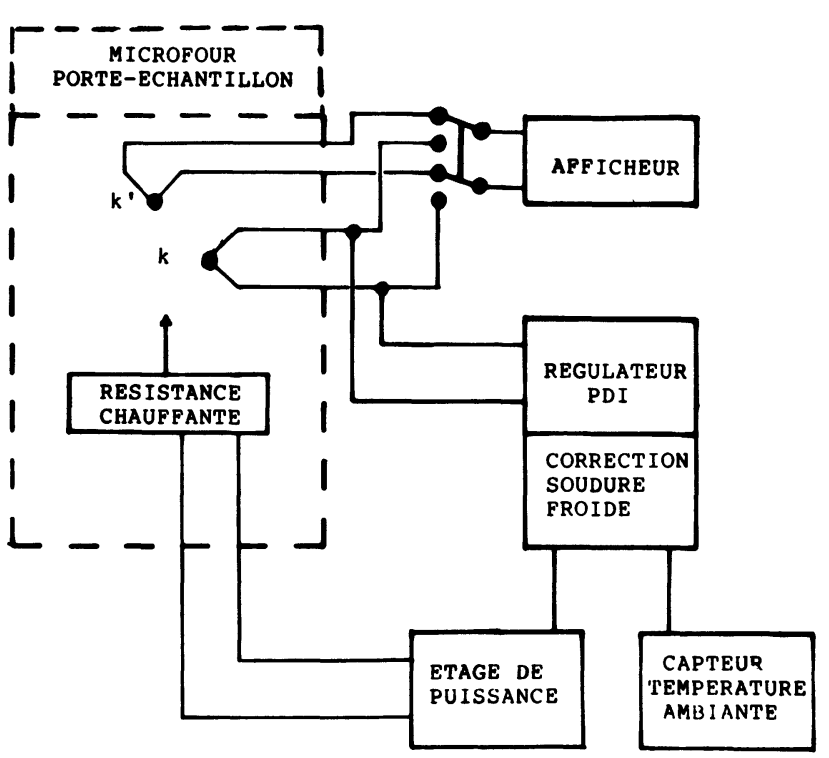

Fig. 4. - Schéma du système de régulation de la température.

[Scheme of the temperature controller.]

Une étude du gradient thermique a été menée dans les conditions suivantes :

- sous un vide de $1 \mathrm{~Pa}$ entre 400 et $1000 \mathrm{~K}$, un gradient radial de $2 \mathrm{~K}$ existe sur l'échantillon. Le gradient au-dessus de l'échantillon est relativement important. La mise en place d'un écran thermique autour du micro-four entraîne cependant une diminution notable de celui-ci ;

— sous hélium $\left(P_{\mathrm{He}}=10^{5} \mathrm{~Pa}\right)$, le gradient radial sur l'échantillon est alors inférieur au degré.

La cellule peut être utilisée soit sous vide, soit avec balayage de gaz divers (neutre, réducteur ou oxydant) dans un domaine de pressions s'étendant de 1 à $2 \times 10^{5} \mathrm{~Pa}$. Le balayage par des mélanges de gaz appropriés permet d'obtenir une large gamme de pressions d'oxygène $\left(10^{-20}\right.$ à $\left.10^{5} \mathrm{~Pa}\right)$. Enfin, compte

REVUE DE PHYSIQUE APPLIQUÉE. - T. 24, N`4, AVRIL 1989 tenu des matériaux utilisés, un comptage de $7 \times$ $10^{5}$ coups par heure peut être obtenu avec une source ${ }^{57} \mathrm{Co}$ de $20 \mathrm{mCi}$.

\section{Application à l'évolution thermique des paramè- tres Mössbauer de la phase $\mathrm{SrFeO}_{2,50}$.}

La phase $\mathrm{SrFeO}_{2,50}$ cristallise dans un système orthorhombique : sa structure est de type brownmillérite $[16,19]$. Elle dérive de la structure perovskite par l'existence de lacunes d'oxygène ordonnées selon des rangées de manière à constituer des couches de tétraèdres. Ces couches alternent avec les couches d'octaèdres du réseau perovskite initial (Fig. 5). Seul le fer (III) est présent.

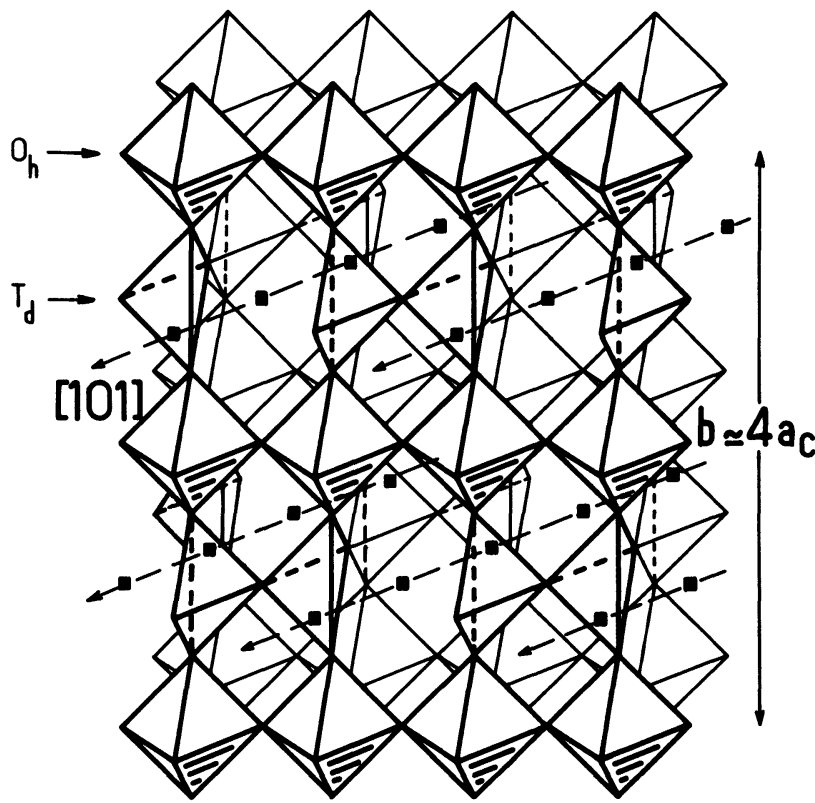

Fig. 5. - Structure idéalisée de la brownmillérite.

[Idealized brownmillerite structure.]

3.1 CONDITIONS EXPÉRIMENTALES. - La composition $\mathrm{SrFeO}_{2,68}$ constitue le produit de départ pour l'obtention des phases $\mathrm{SrFeO}_{3-y}$. Elle est préparée à partir d'un mélange stœchiométrique de $\mathrm{SrCO}_{3}$ et de $\mathrm{Fe}_{2} \mathrm{O}_{3}$. Le mélange finement broyé est porté lentement sous air à $950^{\circ} \mathrm{C}(12 \mathrm{~h})$ puis à $1200^{\circ} \mathrm{C}(6 \mathrm{~h})$ avant d'être trempé. Ce traitement est suivi d'un broyage, d'un recuit à $1300^{\circ} \mathrm{C}$ à l'air en vue d'une homogénéisation et enfin d'une trempe.

La phase $\mathrm{SrFeO}_{2,50}$ est obtenue par recuit sous vide dynamique à $850^{\circ} \mathrm{C}$ de $\mathrm{SrFeO}_{2,68}$ dans un tube de quartz pendant $24 \mathrm{~h}$, suivi d'un refroidissement à $200^{\circ} \mathrm{C} \cdot \mathrm{h}^{-1}$. Le produit recueilli à température ambiante est de couleur brune. La phase a été identifiée par analyse radiocristallographique à l'aide d'une chambre de Guinier.

Les spectres Mössbauer ont été obtenus en utili- 
sant un spectromètre à accélération constante de type Halder utilisant une source ${ }^{57} \mathrm{Co}$ (à matrice de rhodium) maintenue à température ambiante. La masse de l'échantillon pulvérulent est calculée de manière à contenir $10 \mathrm{mg} \mathrm{Fe} / \mathrm{cm}^{2}$. Dans un domaine de températures inférieures à l'ambiante les spectres ont été obtenus à l'aide d'un cryostat à température variable. Entre 293 et $1050 \mathrm{~K}$ ils sont déterminés au moyen d'un dispositif décrit au chapitre précédent sous balayage d'argon purifié $\left(1.1 . \mathrm{h}^{-1}\right)$. L'argon est préalablement séché sur pentoxyde de phosphore ; il traverse ensuite un tube laboratoire de nickel contenant de l'éponge de fer maintenue à $820 \mathrm{~K}$. La pression d'oxygène au-dessus de l'échantillon est alors voisine de $1 \mathrm{~Pa}$ à $823 \mathrm{~K}$.

Les paramètres Mössbauer ont été affinés par la méthode des moindres carrés en supposant des profils de raies lorentziens pour les spectres présentant des raies fines. Pour les spectres obtenus à des températures voisines mais inférieures à $T_{N}$ des effets de relaxation de type superparamagnétique provoquent un élargissement notable des raies d'absorption. Dans ces cas, les paramètres Mössbauer ont été déterminés en utilisant un programme de calcul qui tient compte des effets de relaxation. Tous les déplacements isomériques sont relatifs au fer $\alpha \grave{g} T=293 \mathrm{~K}$.

3.2 Résultats Et Discussion. - Une étude par résonance Mössbauer a été effectuée sur $\mathrm{SrFeO}_{2,50}$ pour le domaine de température $4,2 \leqslant T \leqslant 1050 \mathrm{~K}$. Quelques spectres caractéristiques sont reproduits sur les figures 6 et 7 . Les paramètres Mössbauer sont donnés au tableau I.

En dessous de la température de Néel, les spectres de résonance Mössbauer caractérisent des interactions magnétiques hyperfines. Ils sont constitués de

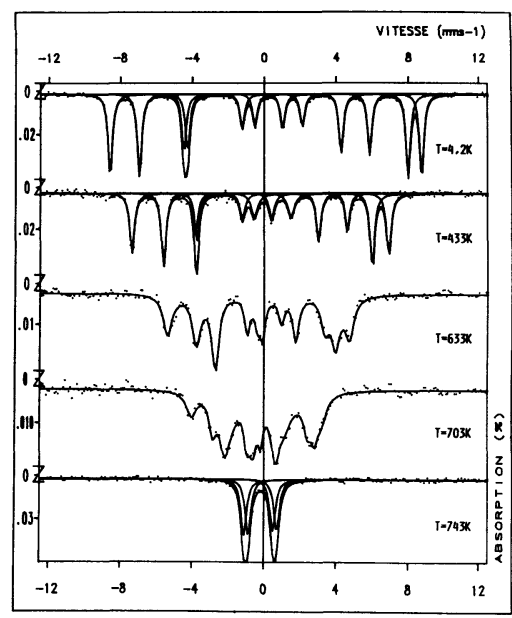

Fig. 6. - Spectres de résonance Mössbauer de $\mathrm{SrFeO}_{2,50}$ $(4,2 \leqslant T \leqslant 743 \mathrm{~K})$.

[Mössbauer resonance spectra of $\mathrm{SrFeO}_{2.50}(4.2 \leqslant T \leqslant$ $743 \mathrm{~K})$.]

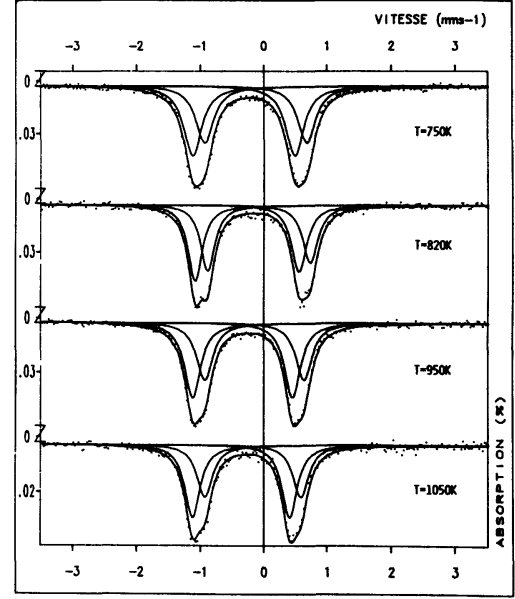

Fig. 7. - Spectres de résonance Mössbauer de $\mathrm{SrFeO}_{2,50}$ $(750 \leqslant T \leqslant 1050 \mathrm{~K})$.

[Mössbauer resonance spectra of $\mathrm{SrFeO}_{2.50}(750 \leqslant T \leqslant$ $1050 \mathrm{~K})$.]

deux sextuplets qui peuvent être attribués au fer trivalent simultanément présent en site octaédrique $\left(\mathrm{O}_{h}\right)$ et en site tétraédrique $\left(\mathrm{T}_{\mathrm{d}}\right)$. Les champs à saturation correspondant à ces deux sextuplets sont $H_{0}\left(\mathrm{O}_{\mathrm{h}}\right)=54 \mathrm{~T}$ et $H_{0}\left(\mathrm{~T}_{\mathrm{d}}\right)=45 \mathrm{~T}$. L'évolution thermique des champs hyperfins est présentée à la figure 8. Compte tenu de la nature des couplages antiferromagnétiques, les deux séries de points expérimentaux ont pu être ajustées par une fonction de

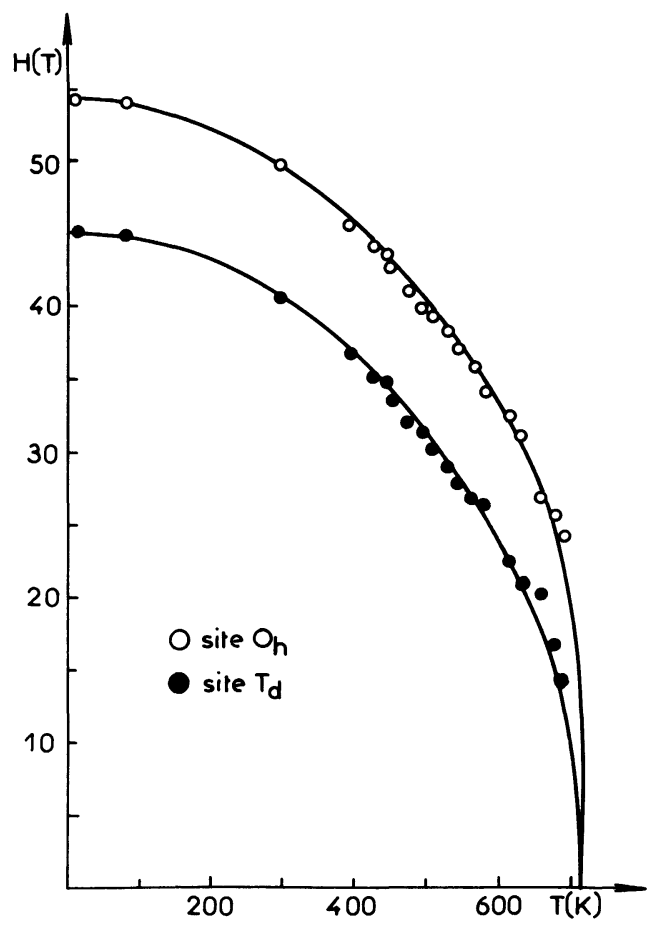

Fig. 8. - Variation thermique des champs hyperfins de $\mathrm{SrFeO}_{2,50}$.

[Thermal variation of the hyperfine fields of $\mathrm{SrFeO}_{2.50}$.] 
Tableau I. - Paramètres Mössbauer de la phase $\mathrm{SrFeO}_{2,50}$.

[Mössbauer parameters of $\mathrm{SrFeO}_{2.50}$.]

\begin{tabular}{|c|c|c|c|c|c|c|}
\hline \multirow{2}{*}{$\begin{array}{c}\text { Températures } \\
(\mathrm{K})\end{array}$} & \multicolumn{2}{|c|}{ Fe (III) en site octaédrique } & \multicolumn{2}{c|}{ Fe (III) en site tétraédrique } \\
\cline { 2 - 7 } & $\begin{array}{c}\delta\left(\mathrm{mm} \cdot \mathrm{s}^{-1}\right) \\
\pm 0,01\end{array}$ & $\begin{array}{c}H(\mathrm{~T}) \\
\pm 0,05\end{array}$ & $\begin{array}{c}\% \\
\pm 1\end{array}$ & $\begin{array}{c}\delta\left(\mathrm{mm}_{\mathrm{.}}-\mathrm{s}^{-1}\right) \\
\pm 0,01\end{array}$ & $\begin{array}{c}H(\mathrm{~T}) \\
\pm 0,05\end{array}$ & $\begin{array}{c}\% \\
\pm 1\end{array}$ \\
\hline 4,2 & 0,49 & 54,00 & 49 & 0,31 & 45,00 & 51 \\
77 & 0,48 & 54,00 & 48 & 0,31 & 44,90 & 52 \\
293 & 0,37 & 49,62 & 47 & 0,21 & 40,75 & 53 \\
433 & 0,26 & 44,07 & 47 & 0,10 & 35,15 & 53 \\
532 & 0,18 & 39,35 & 46 & 0,05 & 28,92 & 54 \\
633 & $\langle 0,17\rangle$ & $\langle 31,00\rangle$ & 46 & $\langle-0,02\rangle$ & $\langle 21,40\rangle$ & 54 \\
703 & $\langle 0,10\rangle$ & $\langle 24,68\rangle$ & 46 & $\langle-0,05\rangle$ & $\langle 16,31\rangle$ & 54 \\
\hline & $\delta\left(\mathrm{mm} . \mathrm{s}^{-1}\right)$ & $\Delta\left(\mathrm{mm} / \mathrm{s}^{-1}\right)$ & $\%$ & $\delta\left(\mathrm{mm} . \mathrm{s}^{-1}\right)$ & $\Delta\left(\mathrm{mm} . \mathrm{s}^{-1}\right)$ & $\%$ \\
& $\pm 0,01$ & $\pm 0,01$ & \pm 1 & $\pm 0,01$ & $\pm 0,01$ & \pm 1 \\
\hline 750 & 0,08 & 1,60 & 45 & $-0,11$ & 1,60 & 55 \\
820 & 0,03 & 1,58 & 45 & $-0,15$ & 1,58 & 55 \\
950 & $-0,03$ & 1,56 & 45 & $-0,21$ & 1,56 & 55 \\
1050 & $-0,07$ & 1,47 & 43 & $-0,25$ & 1,48 & 57 \\
\hline
\end{tabular}

Brillouin en considérant un spin $S=5 / 2$. Le lissage de nos points expérimentaux a conduit aux valeurs $T_{\mathrm{N}}=711 \pm 5 \mathrm{~K}$ et $714 \pm 5 \mathrm{~K}$ respectivement pour le sous-réseau tétraédrique et le sous-réseau octaédrique. La proximité de ces valeurs montre l'existence d'une température de Néel unique pour les deux sous-réseaux. Ce résultat est en accord avec Geller $e t$ al. [20], mais en revanche en désaccord avec celui de Whitfield [21] qui a supposé que les sous-réseaux avaient des températures de Néel différentes. Enfin un bon accord est observé entre la valeur de la température de Néel obtenue par mesures magnétiques et celle déterminée par résonance Mössbauer [16].

Au-dessus du point de Néel $\left(T_{\mathrm{N}} \leqslant T \leqslant 1050 \mathrm{~K}\right)$ les spectres Mössbauer sont constitués de deux doublets relatifs aux atomes de fer en sites $\left(\mathrm{O}_{h}\right)$ et $\left(T_{d}\right)$ (Fig. 7).

A l'encontre de ce qu'on eût pu prévoir le rapport des aires de résonance observées pour les sites $\left(\mathrm{O}_{\mathrm{h}}\right)$ et $\left(\mathrm{T}_{\mathrm{d}}\right)$ n'est pratiquement jamais égal à 1 . Pour certaines températures, l'écart à 1 est même relativement important (Tab. I) bien que la composition après vérification soit bien égale à $\mathrm{SrFeO}_{2,50}$. Ce phénomène ne pouvant donc se justifier par des taux de fer différents dans les sites octaédriques et tétraédriques, il a fallu envisager des fractions sans recul $f$ inégales pour les noyaux de fer au sein des deux types de sites.

Dans l'approximation d'un solide isotrope de Debye la forme complète de la relation exprimant le facteur de Lamb-Mössbauer est :

$$
\begin{aligned}
& f=\exp \{- \frac{3}{4} \frac{E_{\gamma}^{2}}{M c^{2} k_{\mathrm{B}} \theta_{\mathrm{D}}} \times \\
&\left.\times\left[1+4\left(\frac{T}{\theta_{\mathrm{D}}}\right)^{2} \int_{0}^{\theta_{\mathrm{D}} / T} \frac{x \mathrm{~d} x}{\mathrm{e}^{x}-1}\right]\right\}
\end{aligned}
$$

où $E_{y}$ représente l'énergie de la transition nucléaire, $M$ la masse du noyau, $k_{\mathrm{B}}$ la constante de Boltzmann et $\theta_{\mathrm{D}}$ la température de Debye.

La connaissance de la température de Debye pour un noyau Mössbauer donné permet ainsi de calculer l'évolution thermique de $f$.

Le déplacement isométrique $\delta$ de la raie d'absorption Mössbauer est la somme d'une contribution intrinsèque $\delta_{\mathrm{I}}$ et d'une contribution extrinsèque $\delta_{\text {SOD }}$ :

$$
\delta=\delta_{\mathrm{I}}+\delta_{\text {SOD }}
$$

où $\delta_{\text {SOD }}$ est l'effet Doppler du second ordre (second order Doppler effect). Le terme $\delta_{\mathrm{I}}$ est indépendant de la température, alors que $\delta_{\text {SOD }}$ est proportionnel à la vitesse quadratique moyenne du noyau. Pour un solide de Debye ce terme est donné par la relation :

$$
\begin{aligned}
\delta_{\mathrm{SOD}}= & -\frac{9}{16} \frac{E_{\gamma} k_{\mathrm{B}}}{M c^{2}} \times \\
& \times\left[\theta_{\mathrm{D}}+8 T\left(\frac{T}{\theta_{\mathrm{D}}}\right)^{3} \int_{0}^{\theta_{\mathrm{D}} / T} \frac{x^{3} \mathrm{~d} x}{\mathrm{e}^{x}-1}\right] .
\end{aligned}
$$

Pour la phase $\mathrm{SrFeO}_{2,50}$ un programme de calcul utilisant l'équation (3) a permis d'obtenir des courbes 


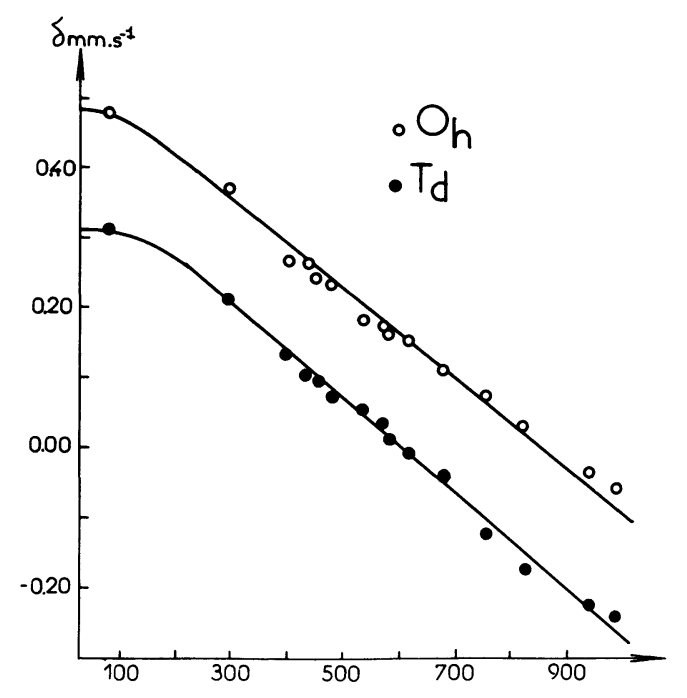

Fig. 9. - Variation thermique des déplacements isomériques de $\mathrm{SrFeO}_{2,50}$.

[Thermal variation of the isomer shifts of $\mathrm{SrFeO}_{2.50}$.]

lissées à partir des points expérimentaux pour les sites $\left(O_{h}\right)$ et $\left(T_{d}\right)$ (Fig. 9). La valeur de la température de Debye relative au site tétraédrique $\theta_{\mathrm{D}}\left(\mathrm{T}_{\mathrm{d}}\right)=514 \mathrm{~K}$ est très supérieure à celle calculée pour le site octaédrique $\theta_{\mathrm{D}}\left(\mathrm{O}_{\mathrm{h}}\right)=425 \mathrm{~K}$.

En reportant ces valeurs dans l'équation (1) on

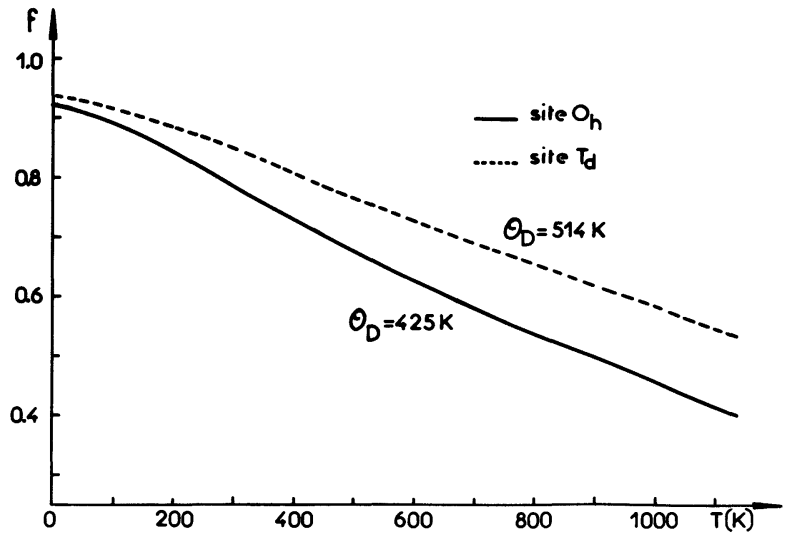

Fig. 10. - Variation thermique des facteurs $f$ pour $\mathrm{SrFeO}_{2,50}$.

[Thermal variation of the $f$ factors for $\mathrm{SrFeO}_{2.50}$.]

peut calculer les facteurs $f$. Dans tout le domaine de température considéré, la fraction sans recul des noyaux de ${ }^{57} \mathrm{Fe}$ dans les sites $\left(\mathrm{T}_{\mathrm{d}}\right)$ est supérieure à celle des noyaux situés dans les sites $\left(\mathrm{O}_{h}\right)$ (Fig. 10). Le noyau $\mathrm{Fe}\left(\mathrm{T}_{\mathrm{d}}\right)$ est certainement plus fortement lié à l'oxygène que le noyau $\mathrm{Fe}\left(\mathrm{O}_{\mathrm{h}}\right)$. Si on tient compte des facteurs $f$, le rapport (taux $\mathrm{Fe}\left(\mathrm{O}_{\mathrm{h}}\right) / \operatorname{taux} \mathrm{Fe}\left(\mathrm{T}_{\mathrm{d}}\right)$ ) à toute température est alors voisin de l'unité en accord avec la composition et la structure cristalline de la phase.

\section{Bibliographie}

[1] Kolk B. et Bleloch A., Rev. Sci. Instrum. 56 (1985) 1597.

[2] VAN DER WOUde F. et BOOM G., Rev. Sci. Instrum. 36 (1965) 800.

[3] Kobeissi M. A. et Hohenemser C., Rev. Sci. Instrum. 49 (1978) 601.

[4] Topsoe H., Dumesic J. A. and Morup S., Application of Mössbauer Spectroscopy (Academic Press) 2 (1980) 57.

[5] Coey J. M. D., Price D. C. et Morrish A. H., Rev. Sci. Instrum. 43 (1972) 54.

[6] Sharon B. et Treves D., Rev. Sci. Instrum. 37 (1966) 1252.

[7] Vaishnava P. P. and Montano P. A., J. Phys. Chem. Solids 43 (1982) 809.

[8] Salomon S., Wallner W. and West J. P., Mössbauer effect Methodology, Eds. J. J. Gruverman et C. W. Seidel (Plenum Press) 10 (1976) 291.

[9] Pijolat M., Thèse d'Etat, Université Claude Bernard, Lyon I (1983).

[10] Gallagher P. K., MacChesney J. B. and BuchaNAN D. N. E., J. Chem. Phys. 41 (1964) 2429.

[11] Tofield B. C., Greaves C. and Fender B. E. F., Mat. Res. Bull. 10 (1974) 737.
[12] Tofield B. C., Reactivity of Solids, Ed. J. Wood (Plenum Press) 1976.

[13] Grenier J. C., Pouchard M. and Hagenmuller P., Structure and Bonding, 47 (Springer-Verlag Berlin Heidelberg) 1981.

[14] TAKano M., NAKanishi N., TAKeda Y. and ShinJo T., Proc. Conf. Ferrites (September-October 1980) Japan.

[15] Gibb T. C., J. Chem. Soc. Dalton trans. (1985) 1455.

[16] Grenier J. C., Ea N., Pouchard M. and HagenMULler P., J. Solid State Chem. 58 (1985) 243.

EA N., Thèse $3^{\text {e }}$ Cycle, Université de Bordeaux I (1983).

[17] MacChesney J. B., Sherwood R. C. and Potter J. F., J. Chem. Phys. 43 (1965) 1907.

[18] Takeda Y., Kanno K., Takada T., Yamamoto O., TAKano M., NAKAYAMA N. and BANDO Y., J. Solid State Chem. 63 (1986) 237.

[19] Bertaut E. F., Blum P. and S^anières A., Acta Crystallogr. 72 (1959) 149.

[20] Geller S., Grant R. W., Gonser U., Wiedersich H. and EspinosA G. P., Phys. Lett. 25 (1967) 722.

[21] Whitfield H. J., Aust. J. Chem. 20 (1967) 859. 